\title{
Quand le PLM délocalise ses dépôts de locomotives... Tonnerre : un destin ferroviaire précaire
}

When the PLM Company relocated its railway depots... Tonnerre: a precarious railway destiny

\section{Georges Ribeill}

\section{OpenEdition \\ Journals}

Édition électronique

URL : https://journals.openedition.org/rhcf/430

DOI : $10.4000 /$ rhcf. 430

Éditeur

Rails \& histoire

\section{Édition imprimée}

Date de publication : 31 mai 2008

Pagination : 87-108

ISSN : 0996-9403

\section{Référence électronique}

Georges Ribeill, «Quand le PLM délocalise ses dépôts de locomotives... Tonnerre : un destin ferroviaire précaire », Revue d'histoire des chemins de fer [En ligne], 38 | 2008, mis en ligne le 10 mai 2011, consulté le 22 avril 2022. URL : http://journals.openedition.org/rhcf/430 ; DOI : https://doi.org/10.4000/rhcf 430 


\title{
Quand le PLM délocalise ses dépôts de locomotives... Tonnerre : un destin ferroviaire précaire
}

\author{
Georges RIBEILL
}

ette contribution veut illustrer les fortes dépendances en-
tre deux logiques territoriales. D’une part, les lignes et ré-
seaux de chemins de fer obéissent à une rationalité complexe, à la fois politique, économique et technique, dont résultent leurs tracés et l'emplacement des gares et des bâtiments de service (dépôts de la traction, ateliers du matériel) ; d'autre part, les villes se développent largement en quartiers et faubourgs en fonction de leurs équipements et accès aux réseaux publics (voirie, gare, transports publics). Chacune de ces logiques a son échelle spatiale et sa dynamique sociale propres, mais lorsque le chemin de fer pénètre dans la ville, leur interférence conduit à des effets structurants très marqués et irréversibles : en général excentrée, la gare appelle à elle la nouvelle « avenue de la gare » qui la reliera au centre ville, tandis que parfois un faubourg cheminot naît « derrière la gare », de l'autre côté des voies ferrées et de leurs dépendances. La problématique classique des «villes-champignons » nées du rail s'est plutôt attachée à apprécier spatialement et démographiquement ces effets d'entraînement. Elle s'intéresse moins en revanche au déclin possible qui peut survenir lorsque un établissement ferroviaire important (dépôt, atelier, triage, etc.), pour une raison ou une autre, voit son activité reportée dans un autre site, voire est « fermé » sans report : délocalisation ou désertification, telles sont les conséquences de cet éventuel coup d'arrêt à la synergie qui lie ville et chemin de fer. L'histoire des chemins de fer en Bourgogne permet de traiter de ce thème, " grandeur et déclin » d’une commune arrimée au 
PLM, à travers deux cas de "villes dépôts ». Le plus connu est bien sûr celui de Laroche-Migennes ${ }^{1}$, dont trois étapes ferroviaires scandent un essor démographique exceptionnel : arrivée du chemin de fer depuis Paris en 1849; ouverture de l'embranchement d'Auxerre en 1855 ; implantation, surtout, d'un dépôt de locomotives en $1880^{2}$. Moins connu est le cas de Tonnerre, selon les points de vue "gare terminus » ou «tête de ligne » du même chemin de fer ouvert en 1849. Tonnerre est doté d'une station classée " gare exceptionnelle », mais aussi d'un dépôt de locomotives avec son atelier de réparation, dépôt transféré justement en 1880 à Laroche.

Notre propos sera centré sur ce «site ferroviaire de première génération ». Dans l'histoire du PLM, Migennes, auquel la fonction de dépôt, décidée trente ans plus tard, assurera un destin imprévu, constitue selon nous en effet par opposition un «site ferroviaire de seconde génération ». Pour être complet, nous avons entrepris l'étude d'un « site ferroviaire de troisième génération ", Les Laumes en Côte-d’Or, nouveau dépôt créé au sortir de la Première Guerre mondiale, 40 ans après celui de Laroche : ainsi, cette approche diachronique ${ }^{3}$ achevée de trois " générations » de dépôts vapeur du $\mathrm{PLM}^{4}$ permettra de comparer leurs impacts territoriaux, économiques et sociaux successifs en Bourgogne ${ }^{5}$. L'existence et l'activité de ces trois dépôts, auxquels il faudrait ajouter Montereau, ont été déterminées par une même logique strictement ferroviaire, leur rationalisation économique, elle-même tributaire des progrès des locomotives dont ces dépôts assurent le ravitaillement en charbon et en eau. Entre Paris et Dijon, cette rationalité technicoéconomique a fixé à Montereau et à Tonnerre deux dépôts intermédiaires en 1849, «fondus » en un seul à Laroche en 1880. Mais

1- Situés sur la commune de Migennes, gare puis dépôt prendront le nom du hameau portuaire près duquel ils seront installés.

2- Nicole Chevalier, «Migennes. Étude urbaine. Les problèmes de la reconversion de cette ville», DES de géographie (sous la dir. de Pierre George), université de Paris, 1962 ; Georges Ribeill, PLM-City... Histoire d'une ville née du rail, Migennes. Du canal au TGV (XIX XX $X^{*}$ siècles), Dixmont, chez l'auteur, 1999.

3- Et non plus de type monographique comme celle qu’ont développée autrefois Yves Baticle et les membres de la commission « histoire des dépôts » de l'AHICF, « Histoire des dépôts de matériel moteur, 1840-1998", Revue d'histoire des chemins de fer, $\mathrm{n}^{\circ} 18$ (printemps 1998).

4- Comparaison facilitée par l'inventaire complet et très fouillé de Marcel Chavy, Olivier Constant, André Rasserie et José Banaudo, Les Dépôts vapeur du PLM, Breil-surRoya, Les Éditions du Cabri, 1997.

5- Un panorama est proposé par Jean Cuynet et Gaby Bachet, Histoire du rail en Bourgogne, Sainte-Croix, Presses du Belvédère, 2007. 
le PLM fera encore mieux en 1919 en créant un dépôt aux Laumes, au pied de la fameuse rampe de Blaisy dont la pente approche 8 pour mille, et en y concentrant les plus puissantes locomotives à vapeur de la « ligne impériale », des 151 dont la seule justification économique est de faire franchir cette rampe aux trains de marchandises les plus lourds.

\section{Trois stations de la "ligne impériale » en perspective}

La comparaison des variations de la population des deux souspréfectures de l'Yonne, Joigny et Tonnerre, avec celle de la commune de Migennes, village agricole transformé en ville moyenne, rattrapant les deux villes précédentes, est suggestive de l'impact du chemin de fer. Si la situation géographique favorable de Joigny, le long de la route nationale 6 et de l'Yonne, avait pu lui faire espérer un important destin de carrefour ferroviaire avec une ligne embranchée sur Montargis et une autre sur Auxerre, le bourg de Migennes, proche de $8 \mathrm{~km}$, lui fut préféré : il était plus proche d'Auxerre, mais aussi et surtout un important carrefour portuaire à la jonction de l'Yonne et du canal de Bourgogne, tout récemment achevé ${ }^{6}$.

L'arrivée de la future « ligne impériale » en 1849 à Migennes (station) et à Tonnerre (station, dépôt et ateliers) s'est accompagnée d'une augmentation significative de leur population entre 1846 et 1851, qui atteignit respectivement $+26 \%$ et $+6 \%$. Mais le transfert du dépôt de Tonnerre à Migennes s'est accompagné de variations encore plus marquées entre 1876 et 1881 : - $10 \%$ d'un côté, + $66 \%$ de l'autre ! Un historien du Tonnerrois a bien montré l'importance du revirement des rapports entre chemins de fer et emplois cheminots :

«C'est à sa situation sur le tracé de la ligne Paris-Lyon-Marseille que Tonnerre doit, au XIX ${ }^{\mathrm{e}}$ siècle, une grande partie de sa notoriété : de 1849 à 1851, notre ville fut même tête de ligne. [...] Siège d'un départ de locomotives, la gare de Tonnerre comptait, vers 1850, plus de 40 agents. Lorsque la ligne de Bourgogne fut totalement mise en service, ce chiffre doubla. Il augmenta encore pour dépasser la centaine quand fonctionnèrent les lignes de Laroche à Auxerre-Clamecy-Nevers, de Cravant aux Laumes et de Clamecy à Cercy-la-Tour. Mais en 1878 le dépôt de Laroche spécialement affecté à l'exploitation de ces embranchements est ouvert ; il en résulte un mouvement de cheminots au détriment de notre ville. Dix ans plus tard, Tonnerre perd le siège de l'inspection principale transférée à Nevers. Aussi, en 1889, quand la compagnie PLM décide de reverser en province la plus grande

6- Voir G. Ribeill, Chroniques migennoises, Première série, Le Port de Laroche à l'âge d'or de la navigation icaunaise, Dixmont, 1995. 
partie des conducteurs en résidence à Paris, la Municipalité présente Tonnerre comme particulièrement bien située pour donner asile à la plupart de ces employés. Hélas la démarche des édiles semble être restée sans effet ${ }^{7}$.»

De 1881 à 1911, alors que Joigny stagne, Migennes gagne 1648 habitants tandis que Tonnerre en perd 1297 (tabl. 1). Si le développement contrasté de leurs trafics et établissements ferroviaires n'est pas seul en cause, le chemin de fer a été pour le moins décisif dans l'essor de $«$ PLM-City $\rangle^{8}$, cité cheminote de seconde génération.

\begin{tabular}{|c|c|c|c|}
\hline Recensement & Joigny & Migennes & Tonnerre \\
\hline 1846 & 6787 & 570 & 4427 \\
\hline 1851 & 6455 & 719 & 4672 \\
\hline 1856 & 6575 & 686 & 4692 \\
\hline 1861 & 5971 & 720 & 4789 \\
\hline 1866 & 6239 & 711 & 5429 \\
\hline 1872 & 6400 & 785 & 5332 \\
\hline 1876 & 6317 & 775 & 5536 \\
\hline 1881 & 6360 & 1288 & 5681 \\
\hline 1886 & 6494 & 1598 & 5095 \\
\hline 1891 & 6218 & 1808 & 4734 \\
\hline 1896 & 6299 & 1895 & 4749 \\
\hline 1901 & 6254 & 2211 & 4685 \\
\hline 1906 & 6057 & 2473 & 4522 \\
\hline 1911 & 6172 & 2936 & 4384 \\
\hline 1921 & 5697 & 3670 & 4373 \\
\hline 1926 & 6610 & 4594 & 4463 \\
\hline
\end{tabular}

Tableau 1. Évolution comparée des populations de Joigny, Migennes et Tonnerre, 1846-1926.

Les évolutions démographiques médiocres de Tonnerre et de Joigny leur coûteront en 1926 leur sous-préfecture, au profit de Sens et d'Avallon, maintenues dans cette fonction.

7- Jean-Pierre Fontaine, Tonnerre et son canton à la Belle Époque, Société d'archéologie et d'histoire du Tonnerrois, 1994, p. 59.

8- Voir G. Ribeill, Chroniques migennoises, Deuxième série, Entre bourg, canal et cité PLM, la naissance d'une ville (1870-1914), Dixmont, chez l'auteur, 2007. 


\section{La bataille régionale des tracés}

Sous la monarchie de Juillet, à l'issue des longues batailles menées pour arrêter le tracé de la ligne de Paris à Lyon (par l'Aube, l'Yonne ou la Nièvre...), Tonnerre eut la chance, inversement à la préfecture Auxerre, de se trouver sur le tracé définitif : la future « ligne impériale » suivrait la vallée de l'Yonne de Montereau jusqu'à Laroche, puis le canal de Bourgogne et l'Armançon, avant de s'engager dans la vallée de la Brenne, atteignant enfin Dijon via le franchissement du Sombernon par le fameux long souterrain pentu de Blaisy ${ }^{9}$. Organisés en de multiples comités locaux, cantonaux, d'arrondissement ou de département, les notables icaunais avaient joué un grand rôle dans cette victoire. Mais entre divers tracés icaunais - par les vallées de la Cure, du Serein ou de l'Armançon -, la concurrence avait joué entre eux, chaque comité prêchant pour sa vallée. Le journaliste et imprimeur Isidore Roze (1796-1883), influent responsable du Tonnerrois, Journal de l'Yonne agricole, commercial et littéraire, secrétaire et délégué de la commission du chemin de fer depuis 1840, milita très activement en faveur du tracé par la vallée de l'Armançon ${ }^{10}$. C'est finalement ce tracé que, le 17 décembre 1843, une commission de députés soumettait au vote de la Chambre, effectivement entériné le 25 juin 1844 (fig. 1).

Restait encore à préciser par le menu le détail de ce tracé. L'un des premiers tracés étudiés par les ingénieurs évoque l'obstacle que constitue la ville de Tonnerre, juchée en hauteur, séparée de l'Armançon par une étroite bande de terrains plats, dégagés et arborés, formant le «Pâtis » : il semblait difficile de bouleverser cet ancestral lieu naturel de promenade des Tonnerrois, en contrebas des étroites et ruelles pentues de la vieille ville ${ }^{11}$. Ainsi, si la ligne, partant de Laroche, se trouvait depuis la ferme de Crécy sur la rive gauche de l'Armançon et au sud du canal, il n'en serait plus de même par la suite à l'approche de Tonnerre, qu'il fallait se résigner à court-circuiter.

9- Sur ces batailles du tracé, voir mes deux ouvrages, De la vapeur aux caténaires. Genèse et essor de la ligne impériale dans la vallée de l'Yonne (1832-1950), Les Amis du Vieux Villeneuve, 1995, chapitre 1, «Une ligne disputée. La bataille des tracés du ParisLyon », p. 3 et sqq. ; PLM-City..., op. cit., chapitre 2, « De la bataille des tracés à la ligne en gestation ", p. 31 et sqq. Sur la mobilisation des Icaunais, voir aussi J. Fromageot, "En marge du $150^{e}$ anniversaire des chemins de fer français », Bulletin de la Société d'archéologie et d'histoire du Tonnerrois, $\mathrm{n}^{\circ} 35$ (1982), p. 68 et sqq.

10- Voir Regnault de Beaucaron, Cent ans à Tonnerre (1783-1883), Macon, Impr. Protat frères, 1939, p. 280, 292, 309, 331, 338.

11- Elie Thiré, « Notes d'histoire sur le Pâtis de Tonnerre », Bulletin de la Société d'archéologie et d'histoire du Tonnerrois, $\mathrm{n}^{\circ} 16$ (1963), p. 11 et sq. 


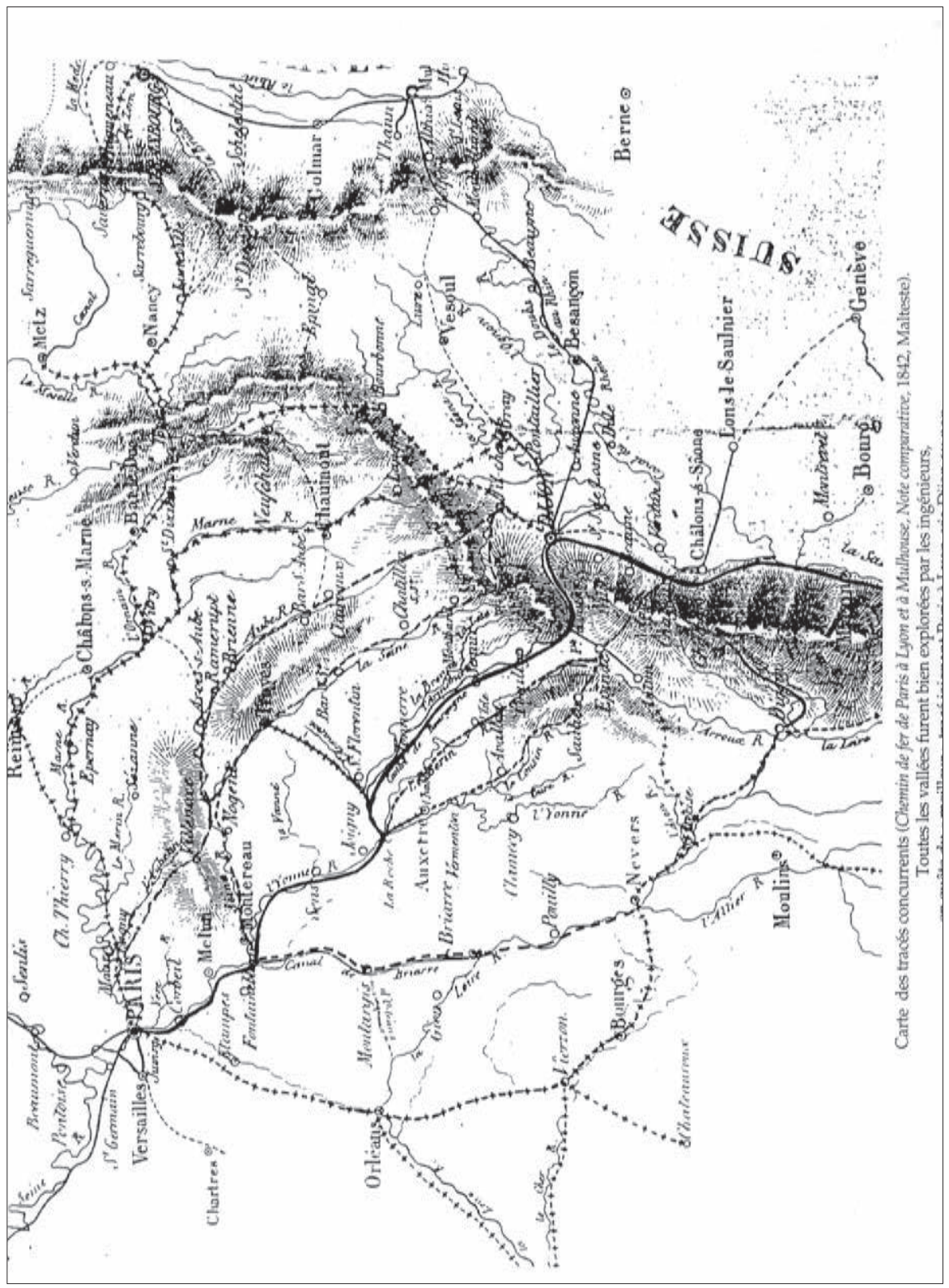


"Ainsi au hameau des Millois, après un passage resserré entre l'Armançon où sont établis plusieurs moulins, et le coteau dont la pente est très rapide, le tracé se retrouve en plaine depuis Roffey jusqu'à Tonnerre, en passant près de la chapelle Saint-Roch et du moulin de Vézinnes, et se reporte ensuite sur la rive droite de la rivière, un peu au-dessus de Junay, pour éviter de traverser la ville ${ }^{12}$. »

Ce n'est que quelques kilomètres plus loin que le tracé rejoignait à nouveau la rive gauche de l'Armançon.

« En approchant de Tonnerre, un premier alignement, parallèle à l'axe du bassin du port, traverse le chemin d'Epineuil et la route royale de Paris à Genève. Trois autres alignements, également parallèles au canal, et partout en remblai, exigent deux fois le redressement de la rivière avant de la traverser pour se reporter sur la rive gauche » [nous soulignons].

L'exiguité du bas quartier de Tonnerre et sa valeur symbolique d'espace de détente avaient motivé ce double franchissement de l'Armançon projeté initialement ${ }^{13}$. Mais c'était oublier les autorités militaires qui avaient leur mot à dire du point de vue de la défense du territoire contre de possibles invasions ennemies venues du Nord-Est. Elles ordonnèrent que les futures voies ferrées soient si possible toujours protégées par les voies d'eau naturelles ou artificielles qui constituaient un barrage naturel à l'avancée des troupes: les chemins de fer aux équipements vulnérables étaient ainsi protégés, tout en servant de rocades facilitant le transport de troupes d'un bout à l'autre de la ligne de front. Reproduites dans une brochure du comité icaunais, deux délibérations du Comité des fortifications (14 avril puis 28 décembre 1841) témoignent de ces préoccupations : la première critiquait un tracé direct de Paris à Strasbourg considéré comme trop proche de la frontière du Nord et donc trop exposé en cas d'invasion; la seconde venait à l'appui du tracé par l'Armançon « sous le rapport stratégique » : en effet, « dans la vallée de l'Armançon, le chemin de fer sera constamment couvert et protégé, soit par les deux cours d'eau, la rivière et le canal, presque constamment à sa gauche »[nous soulignons $]^{14}$.

12- Notice sur le chemin de fer de Paris à Lyon par la Bourgogne, Paris, Impr. Malteste, juillet 1841, p. 41.

13- Il en avait de même sur la ligne au nord de Sens, précisément à Pont-sur-Yonne juché sur un coteau descendant abruptement sur l'Yonne. Là aussi, entre Villemanoche et Sainte-Colombe, au nord de Sens, les premiers plans des ingénieurs faisaient franchir deux fois l'Yonne par la ligne.

14- Chemin de fer de Paris à Lyon et à Mulhouse par Dijon. Note comparative, Paris, Impr. Malteste, mars 1842, p. 10, p. 91. 
Ces brèches potentielles que signifiait ce « presque constamment » formulé en 1841 disparurent finalement des tracés adoptés ${ }^{15}$. Ainsi, en 1844, alors que les conseils d'arrondissement d'Auxerre et d'Avallon revendiquent encore des tracés par les vallées de la Cure ou du Serein, le conseil municipal de Tonnerre met en avant, dans sa délibération du 6 août 1844, l'argument sécuritaire du tracé suivant les deux voies d'eau, Armançon et surtout canal de Bourgogne dont les banquettes constituent un atout que ne peuvent revendiquer les autres voies d'eau.

«Considérant, en se bornant à un intérêt local, au milieu de tant d'autres, qu'il est de toute évidence que la loi a entendu qu'en quittant la vallée de l'Yonne le chemin de fer suivrait la vallée de l’Armançon depuis l'embouchure de cette rivière dans l'Yonne à Laroche jusqu'à Aisy, village situé à l'extrémité de l'arrondissement de Tonnerre, qu'en effet il a été reconnu par toutes les autorités successivement saisies de l'examen des différents tracés proposés qu'indépendamment de toutes autres considérations, le chemin de fer serait très bien défendu par l'Armançon et le Canal de Bourgogne dont les banquettes offrent un rempart continu contre les attaques de l'ennemi ; qu'il était avantageux pour le commerce que ce chemin suivit la direction du Canal... ${ }^{16} »$

Le tracé définitif traversera donc Tonnerre, au prix d'une large amputation du Pâtis où s'imposait l'emplacement de la future station ${ }^{17}$. Cet espace ouvert et central, à forte charge historique, à l'origine un vaste pré concédé en 1212 par le comte Pierre de Courtenay aux habitants de Tonnerre, leur servait de lieu de promenade. Modifié au cours des siècles, grignoté par suite de cessions ou d'usurpations, sa superficie s'était réduite petit à petit ; mais l'établissement de la station sur une large partie allait marquer un tournant dans la structuration spatiale de la vieille ville ${ }^{18}$.

15- Une autre brochure du même comité bourguignon évoque le tracé « sans difficultés sérieuses » reliant Laroche à Buffon "suivant sans discontinuité la rive gauche de l'Armançon" (Chemin de fer projeté de Paris à Lyon par la Bourgogne. Rapport sur la partie du chemin comprise entre Paris et Châlon-sur-Saône, Paris, Impr. Malteste, p. 23).

16- Archives communales, mairie de Tonnerre, comme tout ce qui suit, sauf mention contraire. Les archives y sont remarquablement classées. Nous remercions très vivement le maire de Tonnerre, Raymond Hardy en 2006, pour les facilités de travail qu'il nous a accordées.

17- À Pont-sur-Yonne, au nord de Sens, au prix d'une profonde tranchée, entaillant le coteau et coupant en deux le bourg.

18- Elie Thiré, Les Rues de Tonnerre. Étude historique publiée sous les auspices de la Société d'Archéologie et d'Histoire du Tonnerrois, $2^{e}$ éd., s.d., p. 80-83. 


\section{L'implantation débattue de la station et des ateliers}

À la Compagnie du Paris-Lyon, concessionnaire depuis $1845 \mathrm{de}$ la ligne et en charge de sa construction, il serait facile d'imposer ses vues dans les négociations entreprises avec la ville pour l'acquisition des terrains, non seulement pour la station mais aussi pour ses futurs ateliers, comme nous l'apprend un article de La Presse daté du 19 avril 1846, relatant une décision de la Compagnie du Paris à Lyon capitale quant au destin ferroviaire de Tonnerre.

«Il est décidé provisoirement qu’on ne travaillera cette année que jusqu'à Tonnerre. De prime abord, on voulait pousser les travaux jusqu'à Montbard, mais on a réfléchi et on a vu qu'il était impossible de songer à exploiter la ligne jusqu'à cette dernière ville. Car dans ce cas-là, il aurait fallu établir une grande station provisoire et l'atelier des réparations à Montbard, établissement qui aurait été abandonné par la suite, tandis qu'à Tonnerre, il y aura toujours une station de $1^{\text {te }}$ classe, des grands ateliers et magasins. Voilà donc la raison pourquoi on a résolu d'exécuter d'abord la construction entre Paris et Tonnerre. Quant à la seconde partie depuis Tonnerre jusqu'à Dijon, elle sera commencée l'année prochaine, et elle sera terminée en même temps que le grand souterrain, dans 3 ou 4 ans d'ici. »

En faisant de la ville le terminus provisoire de la ligne de Paris à Dijon, la compagnie installerait à côté du débarcadère un dépôt de locomotives et les ateliers d'entretien attenants... Pour les Tonnerrois, les heureuses retombées économiques et sociales d'un tel projet allaient dès lors l'emporter sur toute autre considération, urbaine ou écologique (fig. 2).

Ouverte du 2 au 10 juin 1846, l'enquête de commodo et incommodo développe les très nombreux arguments en faveur de l'emplacement des installations ferroviaires au Pâtis, répondant tant à l'intérêt général qu'aux intérêts très particuliers des riverains du futur embarcadère.

«Les habitants de la ville de Tonnerre soussignés déclarent approuver dans son entier le tracé adopté par l'ingénieur en chef du chemin de fer M. Jullien, tel qu'il existe au Plan déposé à la mairie de Tonnerre. Ils insistent particulièrement pour que ce tracé ne soit pas changé dans sa partie la plus rapprochée de la Ville, où ce tracé, après avoir traversé la propriété des Minimes, coupe diagonalement la promenade du Pâtis, traverse le marché aux chevaux et la propriété de M. Rendu.

Il importe en effet à l'intérêt bien entendu de la généralité des habitants de Tonnerre que le chemin de fer soit établi le plus près possible de la ville. Les relations nouvelles et multipliées qui doivent exister entre ce centre d'industrie et les habitants, les besoins 


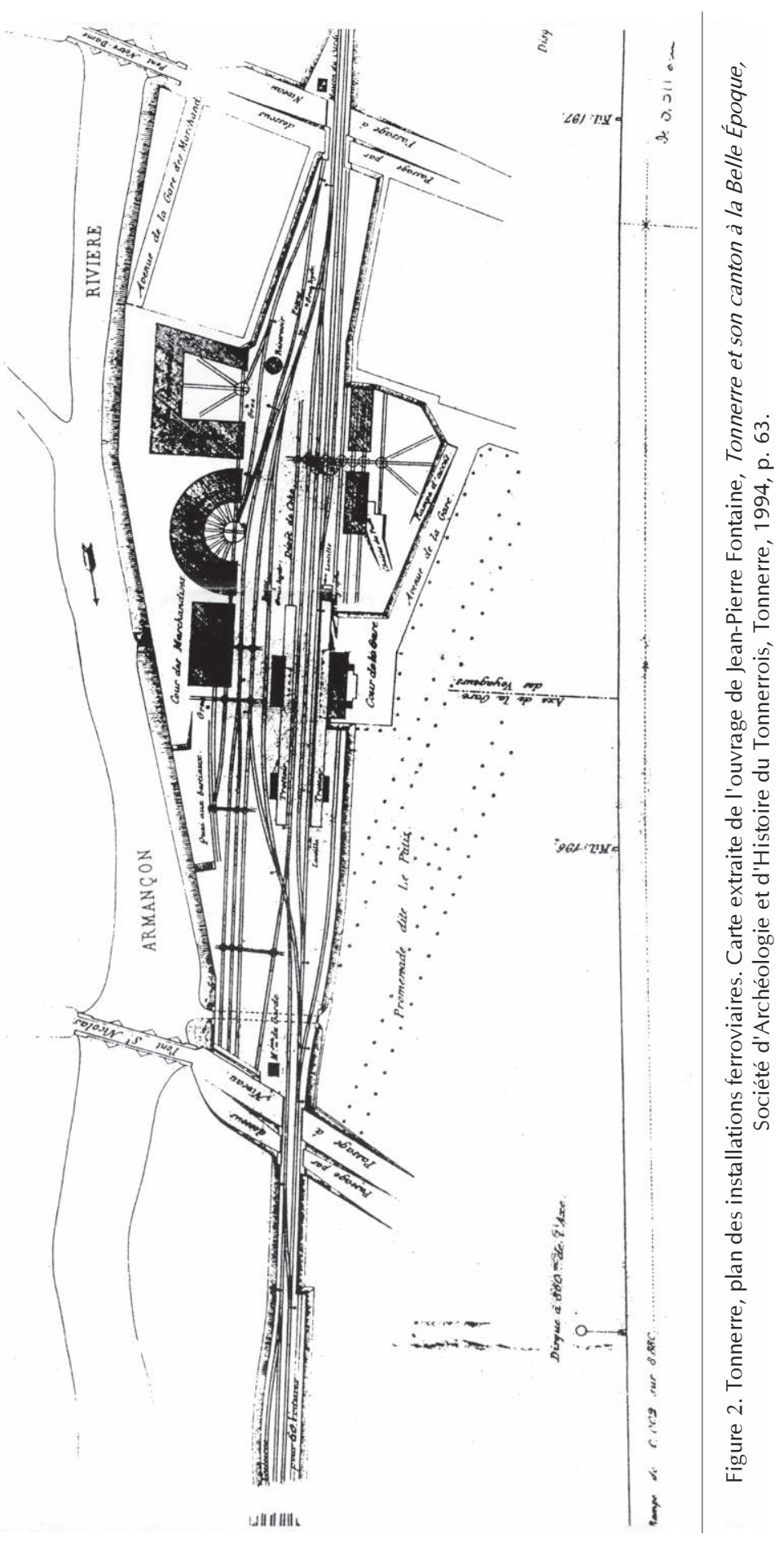


nouveaux qui seront créés, indiquent l'avantage incontestable qu'il y aura pour tous en rapprochant le chemin de fer des habitations. Il serait extraordinaire quand toutes les autres villes de France qui sont dans les mêmes conditions, demandent que les voies de fer les traversent dans leur parcours, de voir les habitants de Tonnerre s'éloigner seuls de cette loi, et vouloir rejeter comme un inconvénient ce que toutes les autres regardent comme un avantage. Traversant le Pâtis qui est aujourd'hui une promenade déserte à cause de son insalubrité, le chemin de fer en laisse une portion notable et suffisante qu'il assainit et rend à la vie et au mouvement.

Placé entre la rivière de l'Armançon et la ville de Tonnerre, la voie de fer aura l'avantage de protéger à l'avenir des inondations la partie basse de la Ville qui n'en était pas toujours à l'abri.

Enfin un avantage que tout citoyen désintéressé et loyal reconnaîtra, c'est que le chemin de fer placé dans le lieu indiqué par le tracé et touchant la ville qu'il longe sans la morceler, ne déplace aucune valeur, ne lie aucun droit acquis. Personne ne met en doute que, si le chemin de fer était éloigné de la ville, par exemple placé de l'autre côté de l'Armançon à côté du canal, les rapports nouveaux avec l'embarcadère à établir pendant le temps où le chemin de fer sera tête de ligne et pendant le temps ultérieur, tout le commerce de la Ville, tout ce qui est actif et vivace se transporterait aux abords du chemin de fer, couvrirait la plaine et créerait une seconde ville au grand détriment de tous, même de ceux qui suivraient avec des dépenses nouvelles le chemin de fer. Toutes les valeurs seraient déplacées, ce serait une perturbation énorme, incalculable dans les intérêts généraux ; tandis que si le chemin de fer s'adosse à la ville, rien n’est déplacé et les propriétés de chacun conservent leurs valeurs. »

Le 13 juin 1846, lors de la séance consacrée à l'examen des résultats de l'enquête, si quelques conseillers municipaux proposent de « rejeter la voie sur la rive droite de la rivière », ils ne peuvent réfuter les nombreux « considérants » votés par la majorité municipale, dont le premier était ainsi formulé :

«Considérant que le tracé par le pâtis, outre un emplacement magnifique pour le débarcadère et les ateliers du chemin de fer, présente l'avantage de l'asseoir sur un terrain plus solide et plus élevé, de diminuer, pour le faubourg du pont et les autres parties basses de la ville, les effets des grandes inondations par le barrage qu'il établira sur la rive gauche du bief du moulin et dans le pré de l'hospice, de maintenir l'unité de la ville, de conserver aux propriétés de l'intérieur leur valeur actuelle et aux diverses industries l'espoir de toutes les améliorations qu'elles sont en droit d'attendre... » 
Le conseil municipal se rallia donc au tracé à travers le Pâtis et le marché aux fourrages adjacent retenu par la compagnie, à moins que de nouvelles études ne démontrent la possibilité de le rapprocher plus encore de l'Armançon, "laissant ainsi à la promenade du Pâtis une surface plus grande et plus régulière ». La compagnie ne fit aucune étude complémentaire à ce sujet, au contraire. Le 27 décembre 1846, informé « des bruits qui circulent » concernant l'intention de la compagnie « de placer ailleurs ses établissements », le conseil municipal invoque le contrat implicite : «La ville a toujours compté et dû compter sur le placement au Pâtis de la station principale du débarcadère et des ateliers, comme un dédommagement de la privation résultant de ce tracé d'une partie importante de la promenade du pâtis. » Mais cela ne suffit pas à le rassurer quant aux bonnes intentions de la compagnie, à laquelle il lui concède donc une nouvelle faveur, " l'abandon gratuit des terrains improductifs du Pâtis formant l'emprise des établissements du chemin de fer ».

Ainsi, le conseil municipal s'était résigné en fin de compte à déplacer et à réduire la place dévolue aux marchés aux fourrages et aux chevaux, à amputer largement les promenades du Pâtis plantées de marronniers et de tilleuls, à faire disparaittre une très utile blanchisserie jouxtant l'Armançon, tous bouleversements conduisant à l'actuelle configuration du Pâtis ${ }^{19}$. En 1847, la compagnie versa tout de même 36000 francs à la commune pour la cession de ses emplacements affectés à ses deux marchés aux fourrages et aux chevaux. $\mathrm{Si}$ « pour avoir les ateliers du chemin de fer ", le conseil municipal unanime avait abandonné une large fraction des terrains du Pâtis, "sacrifice irréparable », jugeait alors Roze dans Le Tonnerrois du 17 janvier $1847^{20}$, les Tonnerrois pouvaient du moins se réjouir de disposer sûrement et prochainement d'une grande station. Au carnaval de 1849, quelques mois avant l'ouverture de la ligne, « le chemin de fer fit sensation sous la forme d'un ogre Vulcain commandant et dirigeant une locomotive monstre trainée par des cyclopes au bruit de fifres et de tambours $»^{21}$.

\section{Enserré au cœur du vieux Tonnerre, un complexe ferro- viaire et ses agents}

Le tronçon de Paris à Tonnerre fut inauguré le 9 septembre 1849, le président de la République Louis Napoléon Bonaparte se rendant simplement à Sens où se déroulèrent de majestueuses cérémonies ${ }^{22}$. Le

19- Elie Thiré, «Note d'histoire... », art. cit., p. 18.

20- Regnault de Beaucaron, op. cit., p. 379.

21- Jean Fromageot, art. cit., p. 74.

22- G. Ribeill, De la vapeur aux caténaires, op. cit., p. 39-41. 
journal L'Illustration jugea pertinent d'illustrer son reportage par une gravure représentant une locomotive haut-le-pied dans les emprises de la station-terminus de Tonnerre, séparée par le fameux Pâtis arboré de l'église Saint-Pierre haut perchée : la confrontation entre l'ancien et le moderne au cœur de la ville, sobrement symbolisée, était fort bien traduite par cette image (fig. 3).

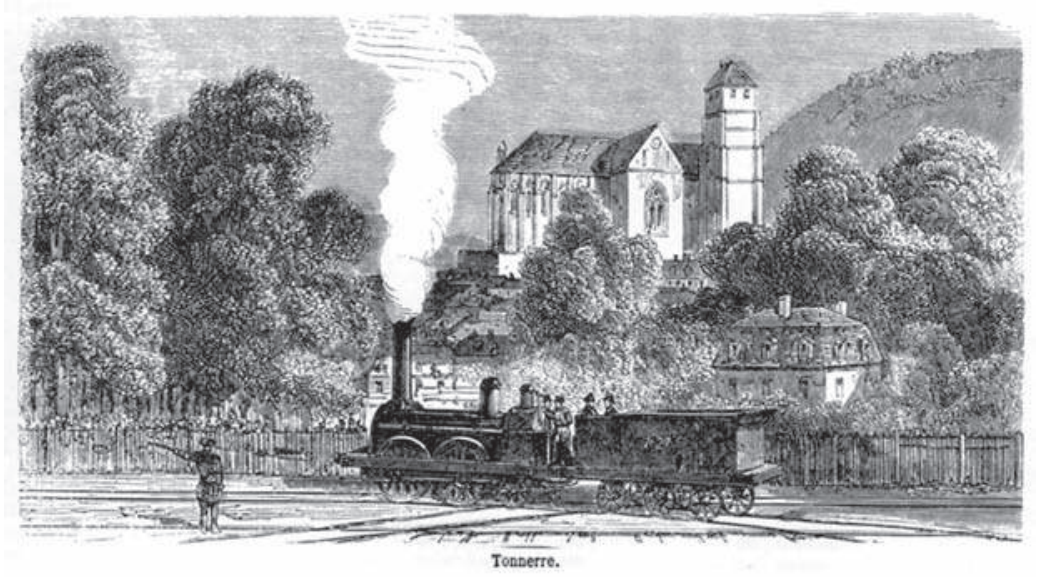

Figure 3. Une locomotive 021, circulant haut-le-pied à Tonnerre, L'Illustration, 15 septembre 1849.

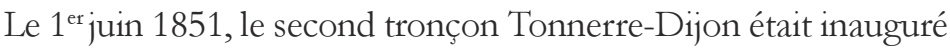
à son tour. Lors des cérémonies étalées sur deux jours, Louis Napoléon Bonaparte s'arrêta avant son arrivée à Dijon à Tonnerre dans la matinée pour y passer en revue les gardes nationales de l'arrondissement: la célébration de son passage et l'inauguration de la ligne achevée furent prétexte à une journée de festivités mêlant revue de troupes, salves d'artillerie, sonneries de cloches, pavoisement de la promenade, jeux publics, bal champêtre sur le Pâtis illuminé, le tout clôturé par un feu d'artifice commandé à Ruggieri.

Comme Montereau, aux fonctions similaires, le dépôt de Tonnerre accueillit en 1849 des locomotives de type 111 pour assurer le relais de la remorque des trains express de voyageurs, autorisés à la vitesse de $80 \mathrm{~km} / \mathrm{h}$. Viendront par la suite des Crampton type 210, des 030 Mammouth, des 021 aussi, le trafic marchandises étant confié à des 030 type Bourbonnais ${ }^{23}$. Une demi-rotonde construite entre les voies ferrées

23- Marcel Chavy, et al., op. cit., p. 281. 
et l'Armançon, des ateliers, une halle à marchandises complétaient le bâtiment voyageurs qui, dans la hiérarchie des modèles standardisés de gares de la compagnie, eut droit à la plus haute distinction, "gare exceptionnelle », et non pas « station de $1^{\text {re }}$ classe » comme Sens, Joigny ou Laroche. Pas de quoi émouvoir toutefois les deux archéologues icaunais Gustave Cotteau et Victor Petit qui, en 1855, dans leur périple à travers le département, ont dédaigné cette station toute neuve et son dépôt de locomotives, fort étrangers, il est vrai, à leurs préoccupations archéologiques.

«Bien que la station du chemin de fer établie à Tonnerre soit l'une des plus importantes de la ligne, nous ne nous y arrêterons pas, parce que, tout en reconnaissant la beauté et la grandeur des bâtiments, l'archéologie n'a rien à y voir quant à présen $t^{24}$. "

En 1852, parmi les 45 stations qui jalonnent la ligne achevée de Paris à Lyon, le classement par leur recette fait apparaittre Tonnerre au $7^{\mathrm{e}}$ rang avec une recette de 390000 francs, après Paris $(5,540$ millions de francs), Chalon-sur-Saône (2,165 MF), Dijon (0,711 MF), Montereau (0,638 MF), Lyon (0,417 MF) et Joigny (0,416 MF). Heureux débuts, mais classement provisoire bien sûr!

L'impact social et démographique du complexe ferroviaire sur Tonnerre est certain. Le 22 novembre 1850,

«Considérant que depuis longtemps des plaintes s'élèvent contre la malpropreté de la promenade du Pâtis et de ses abords, Considérant que cette malpropreté est le résultat du dépôt d'ordures que les nombreux ouvriers et employés attachés au chemin de fer et l'administration du roulage et des Messageries, laissent continuellement soit aux pieds des arbres, soit aux alentours de la promenade,

Que, déjà, le Conseil municipal frappé des inconvénients qui en résultent tant pour les habitants qui fréquentent la promenade que pour les voyageurs qui attendent le départ des convois, a dans sa séance du 17 mai 1850 appelé l'attention de l'Administration du chemin de fer, en sollicitant l'établissement de lieux d'aisance au Pâtis... »,

le maire doit prendre un arrêté instituant un poste de "gardien du pâtis », assermenté chargé de la surveillance et de la propreté des promenades.

L'examen de deux recensements de la population (1851 et 1876) révèle le saupoudrage des agents du chemin de fer dans tous les quartiers centraux de la ville entourant le Pâtis et ses emprises ferroviaires.

24- Annuaire historique du département de l'Yonne, Auxerre, 1855, p. 463. 
En 1851, 58 ménages d'agents se répartissent ainsi dans les quartiers du Pâtis (18), du Pont (14), de la Fosse Dionne (11), au Faubourg Bourberault (8) ou dans la rue des Tanneries (7). En 1876, 154 ménages sont maintenant plutôt concentrés dans le Faubourg Bourberault (62), les autres épars tout autour ${ }^{25}$. Il est impossible de distinguer leur spécialité professionnelle ou d'apprécier une éventuelle ségrégation géographique, les recenseurs ou recensés s'en tenant le plus souvent à l'appellation «employé du chemin de fer » pour les ou se désigner. Mais, à l'évidence, il a fallu que ces employés et ouvriers trouvent à se loger au cœur de la ville, dans ses vieilles maisons ou, plus facilement, dans les faubourgs rayonnants en extension. Cette dissolution de la population cheminote dans l'étroite ville détonne en comparaison du cas de bon nombre d'autres villes où la gare, souvent excentrée, avait créé autour d'elle un nouveau quartier, celui des cheminots. Tonnerre est aux antipodes de ce modèle avec sa gare et son dépôt enserrés au bas de la vieille ville (fig. 4).

\section{Un site ferroviaire condamné par son exiguïté}

Le vice originel et imprévu du site ferroviaire de Tonnerre réside dans son périmètre restreint, sans possibilité d'extension future, tant il était enclavé de tous côtés par de fortes frontières : au Nord, l'Armançon, au Sud la promenade du Pâtis et, de part et d'autre, deux artères majeures conduisant par delà deux ponts sur l'Armençon et le canal de Bourgogne à la route nationale 5 et à ses embranchements sur Epineuil et Troyes.

Dès 1851, l'ingénieur des ponts et chaussées Chaperon, en charge de la $2^{\mathrm{e}}$ section du Chemin de fer de Paris à Lyon ${ }^{26}$, réclamait à la ville de Tonnerre une bande de terrain du côté du Pâtis pour y installer une nouvelle voie de garage pour les trains de marchandises ; sa plate-forme, construite en remblai et d'une longueur de 90 mètres, serait soutenue par un mur dont l'ingénieur propose un aménagement esthétique.

« Je ne dissimule pas ce que ce mur pourra avoir de disgracieux pour l'aspect de la promenade, et peut-être serait-il préférable de conserver un talus gazonné avec haie vive en occupant sur le Pâtis une bande de terrain de 3 mètres de largeur du côté de la gare de voyageurs, sur une longueur de 44 mètres. »

25- La Fosse Dionne (31), le Pont (24), les Tanneries (17), le Pâtis (12), ou les Guérites (8). 26- Rappelons à l'occasion que la ligne impériale fut exploitée depuis son ouverture en 1849 jusqu'à fin 1851 par l'administration des ponts et chaussées, suite au rachat par l'État en août 1848 de la première Compagnie du Paris-Lyon tombée en déconfiture. Le PLM résulte en 1857 de la fusion d'une seconde Compagnie de Paris-Lyon avec une Compagnie de Lyon à la Méditerranée. 


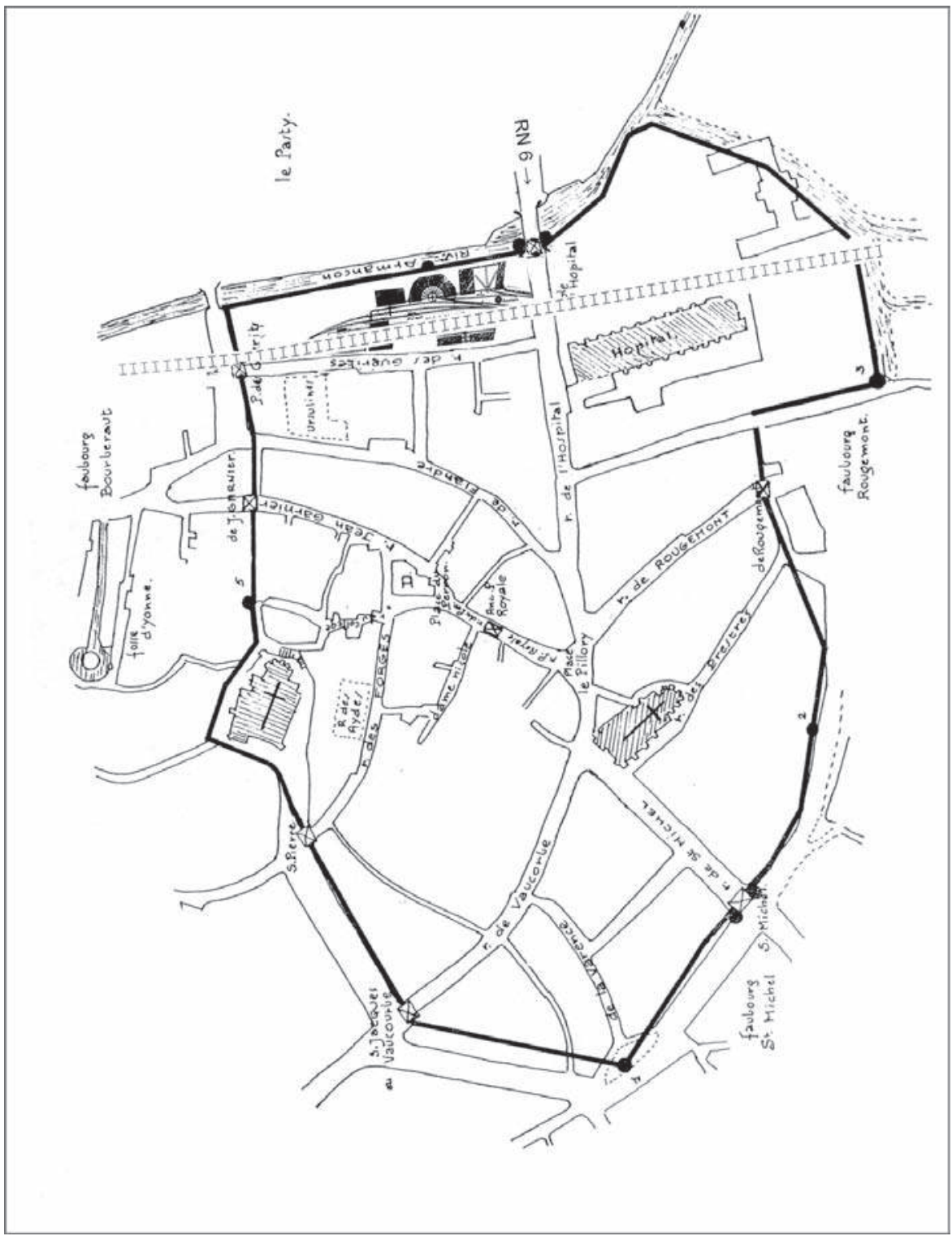

Figure 4. Plan de Tonnerre au XIXe siècle, avec le tracé des murailles de la vieille ville. Fonds de carte extrait de Elie Thiré, Les Rues de Tonnerre. Étude historique publiée sous les auspices de la Société d'Archéologie et d'Histoire du Tonnerrois, 2e éd., s.d., p. 49.

Dessin des infrastructures ferroviaires, G. Ribeill. 
À l'évidence, Tonnerre ne pouvait ainsi connaitre un grand destin ferroviaire. Le développement du réseau du PLM dans le département va renforcer au contraire très tôt la station de Laroche : l'embranchement d'Auxerre y est ouvert en 1855, prolongé sur Clamecy et sur Avallon en 1870 et 1873 ; ces lignes étant elles-mêmes prolongées d'Avallon aux Laumes en 1876, de Clamecy à Nevers en 1877 (fig. 5).

Si c'est en 1874 qu'est connu des élus de Migennes le projet du PLM d'y transférer à moyen terme le dépôt de machines de Tonnerre, c'est seulement à partir de 1877 que cette sombre perspective pousse le conseil municipal de Tonnerre à se mobiliser pour limiter son impact social et économique, ou pour obtenir quelques compensations du PLM. Ainsi, le 31 août 1877, le conseil municipal débat d'un projet de ligne reliant Auxerre à Troyes : ce tronçon d'une plus vaste "ligne stratégique » qui relierait Bourges à la frontière, via Châlons-Sur-Marne et Verdun, en passant de manière obligée par deux "points-force ", Chablis et Ervy-le-Châtel, met en compétition deux tracés officiels intermédiaires, par Saint-Florentin et par Flogny. Mais pourquoi donc pas par Tonnerre, un peu plus à l'Est? Les arguments avancés sont fondés : alors que, entre Chablis et Ervy, les trois tracés ont des longueurs comparables (40 km par Saint-Florentin, $36 \mathrm{~km}$ par Tonnerre, $33 \mathrm{~km}$ par Flogny), primo, le tracé par Saint-Florentin ferait quelque peu double emploi avec l'embranchement d'Auxerre à Laroche ; secundo, « la ligne par Tonnerre serait au contraire à peu près médiane entre celle de Laroche et celle de Nuits à Châtillon dont l'importance stratégique a été constatée dans la dernière guerre. Cette situation permettrait, le cas échéant, de concentrer plus rapidement des troupes sur la frontière de l'Est puisqu'à partir de Tonnerre, elles pourraient, sans perte de temps, être dirigées, suivant les besoins, soit sur Troyes, Châlons et Verdun, soit sur Nuits, Châtillon, Chaumont et Langres »; tertio, Tonnerre, chef-lieu d'arrondissement, est « en état d'infériorité notoire » comparé à Sens, Joigny et Avallon, puisque tout déplacement à Auxerre suppose un long détour avec transbordement en gare de Laroche ; enfin, du point de vue trafic, le tracé traverserait un riche vignoble et une ville beaucoup plus importante que Flogny ou Saint-Florentin, et «le mouvement commercial serait beaucoup plus actif entre les villes d'Auxerre, Tonnerre et Troyes ».

Ce vœu réitéré restera lettre morte, comme en réalité le projet de liaison directe entre Auxerre et Troyes. Exaucé, il aurait assuré à l'évidence un rebond à Tonnerre qui, devenu un important carrefour ferroviaire, aurait conservé un important parc de locomotives en service ordinaire et en réserve stratégique. 


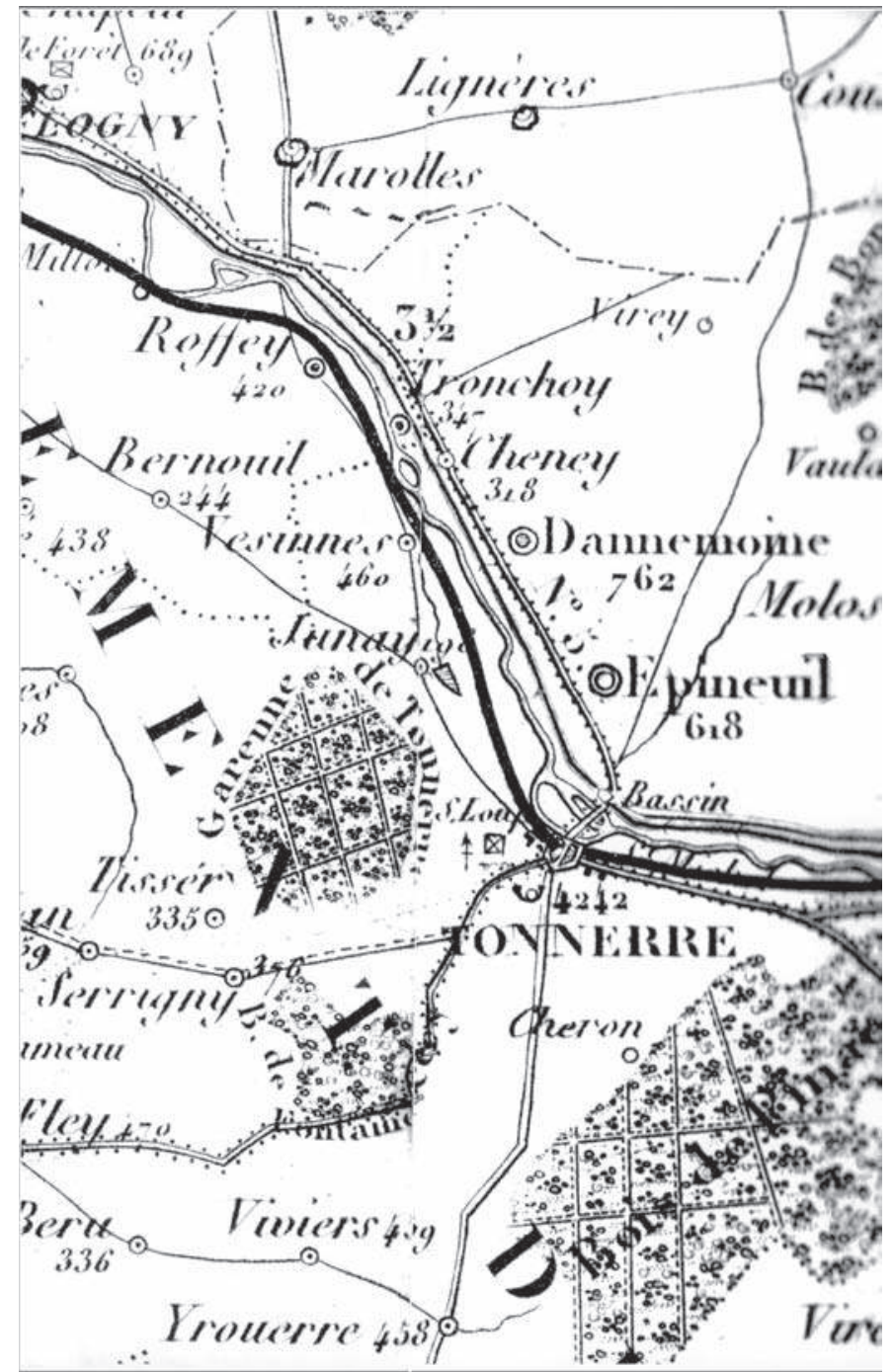

Figure 5. Les voies de communication dans le Tonnerrois. Carte toilée, Département de l'Yonne, extrait de Donnet, Frémin et Levasseur, Atlas des départements de la France, A. Logerot éditeur, s.d.

D'Ouest en Est : la ville, la voie ferrée, les bras de l'Armançon, le canal de Bourgogne et la route nationale. 
Le 13 mai 1878, le conseil délibère explicitement pour la première fois sur la perte du dépôt qui le menace :

«Considérant que la Ville de Tonnerre a cédé gratuitement au chemin de fer 3 hectares 29 ares 40 centiares de terrains pris sur sa promenade du pâtis,

Qu'elle l'a fait à la condition spéciale que la station principale, le débarcadère et les ateliers de réparation y seraient établis, et avec la réserve la plus expresse que la ville pourrait reprendre lesdits terrains dans le cas où, par suite de modifications ultérieures, ils cesseraient d'avoir la destination pour laquelle ils seraient cédés (délibération du 27 décembre 1846) [...],

Considérant que pleins de confiance dans ces conventions, plusieurs habitants de la ville ont fait des constructions dans le but de loger les nombreux employés du chemin de fer appelés à Tonnerre par l'établissement de la station principale, des ateliers de réparation et de l'Inspection, que leur départ porterait un grand préjudice à la Commune,

Invite le Maire à rappeler à la Compagnie de chemin de fer les conventions arrêtées entre elle et la ville, à l'inviter à s'y conformer dans la mesure du possible et dans tous les cas, de lui donner des compensations qu'il se réservera d'apprécier. »

Dans sa réponse au maire, le 19 juillet suivant, le directeur de l'Exploitation du PLM, Bargmann, fait référence au développement qu'a connu le réseau depuis 1849 pour justifier le déplacement du dépôt, et présenter sous une forme habile les pertes d'emploi peu élevées induites par le transfert prévu:

«Vous m’avez fait l'honneur de m’écrire, le 20 juin dernier, pour me transmettre une délibération par laquelle le Conseil municipal de Tonnerre réclame contre la translation à Laroche d'une partie du personnel résidant actuellement à Tonnerre.

Le personnel du dépôt de Tonnerre se composait à l'origine de 40 agents. Par suite du développement du trafic et de l'ouverture complète de la ligne de la Bourgogne, ce nombre a atteint 75 à 80. Les lignes de Laroche à Auxerre, Clamecy et Nevers, de Cravant aux Laumes et de Clamecy à Cercy-la-Tour ayant été ouvertes avant la construction du dépôt de Laroche, qui devait être spécialement affecté à l'exploitation de cet embranchement, nous avons été obligés de placer temporairement à Tonnerre le matériel et le personnel nécessaires au service de ces lignes; mais cette situation ne pouvait être que temporaire, car le dépôt de Tonnerre n'est ni établi ni placé dans des conditions à pouvoir assurer régulièrement le service des lignes du Centre. 
L'ouverture du dépôt de Laroche, qui mettra fin à cette situation toute provisoire, réduira sans doute l'importance actuelle du dépôt de Tonnerre; mais le chiffre du personnel restera sensiblement le même qu'avant l'ouverture des lignes du Centre. » [Nous soulignons.]

Le 5 août suivant, en des termes laconiques, le maire fait part au conseil de cette sorte de « plan social» minimal promis par le directeur du PLM : «Le personnel du dépôt de Tonnerre se trouvait au moment de l'ouverture de la ligne du chemin de fer de 40, et ce chiffre sera toujours conservé malgré l'ouverture du dépôt de Laroche. »

La politique de rationalisation des dépôts du PLM prendrait donc bien appui en définitive sur Laroche : alors que Tonnerre conservait quelques 030 Bourbonnais ${ }^{27}$, le dépôt de Laroche, inauguré le $1^{\text {er }}$ avril $1880^{28}$, recevait la plupart de ses autres locomotives et, dans leur sillage, allait accueillir, dans sa cité toute neuve, les équipes de mécaniciens et chauffeurs titulaires qui les servaient.

\section{Tonnerre en quête de compensations au transfert de son dépôt}

Deux documents suggèrent une quête de compensation de la part des élus. En premier lieu, le 5 février 1880, un projet d'agrandissement du buffet, en face du bâtiment voyageurs, est arrêté par les ingénieurs du PLM : au lieu des 35 couverts qu'autorise l'actuelle table d'hôte, il devra pouvoir assurer 100 couverts $^{29}$. De l'autre côté des voies principales, vis-à-vis du bâtiment voyageurs, cet imposant buffet, avec chambres aux étages, sera bien construit: il est encore visible aujourd'hui mais désaffecté. Trois ans plus tard, les élus essaient d'attirer à Tonnerre des agents parisiens que la compagnie a décidé de rapatrier en province. Ainsi, le 9 mars 1883, le maire s'adresse ainsi au directeur de l'Exploitation :

« Le conseil, ayant eu connaissance que la Compagnie Paris-Lyon venait de décider de reverser en province la plus grande partie des conducteurs-chefs et conducteurs [agents des trains, à ne pas confondre avec les mécaniciens et les chauffeurs], en résidence actuellement à Paris, Considérant que Tonnerre se trouve dans d'excellentes conditions pour donner asile à la plupart de ces employés, attendu que la gare se trouve presque dans le centre de la ville et favorise par

27- M. Chavy, et al., op. cit., p. 281. Sur l'histoire plus générale des dépôts, voir Yves Baticle, monographie citée.

28- M. Chavy, et al., op. cit., p. 129.

29- Archives de la Région Paris - Sud-Est de la SNCF, Bâtiments, Archives municipales de Tonnerre. 
conséquent le service que la Compagnie peut exiger d'eux ; qu'ensuite cette ville est admirablement située pour fournir à tous les besoins de ces agents,

Considérant, d'autre part, que la ville a éprouvé un préjudice réel en raison du départ récent d'un grand nombre d'employés des chemins de fer de l'inspection principale transférée maintenant à Nevers,

Par ces motifs, émet le vœu ${ }^{30}$ qu'il soit demandé à la Compagnie PLM de vouloir bien prendre en considération la situation exceptionnelle de la ville et reverser à Tonnerre la plus grande partie des employés dont elle pourra disposer,

J'ai l'honneur de vous prier M. le Directeur, de vouloir bien l'appuyer auprès de la Compagnie qui voudra bien, j’ose l'espérer, faire droit à cette demande, dans la mesure du possible. »

Espoir déçu, suite à la réponse du sous-directeur de la Compagnie, Coffinet, le 21 mars :

«Monsieur le Maire,

Vous m'avez fait l'honneur de m'écrire, le 9 de ce mois, pour me transmettre un vœu du Conseil municipal de votre ville tendant à obtenir que notre Compagnie assignât la résidence de Tonnerre à la majeure partie des conducteurs de trains auxquels elle allait faire quitter Paris.

La mesure à laquelle cette demande fait allusion n'a pas, à beaucoup près, l'importance qu'on lui attribue. Nous avons, en effet, dans ces derniers temps, déplacé de Paris quelques conducteurs dont la résidence n'était pas bien choisie eu égard aux exigences du service ; il s'agissait de trains du Bourbonnais, et les conducteurs dont il s'agit, ont dû être envoyés à Nevers.

Je ne pense pas que nous ayons à opérer d'autres déplacements d'agents des trains.

Quoi qu'il en soit, je prends bonne note du désir dont vous avez bien voulu vous faire l'interprète et ne manquerai pas d'y donner telle satisfaction que les circonstances pourront comporter dans l'avenir.

Veuillez agréer, Monsieur le Maire, l'assurance de ma considération distinguée. »

On reconnait dans ces diverses lettres la froideur coutumière des dirigeants du PLM sans la moindre compassion à l'égard des édiles locaux, insensibles à leurs préoccupations sociales et strictement mus par les calculs de rentabilité de leurs investissements. Ainsi la logique économique

30- Rayé sur le brouillon de la lettre : «que la Compagnie prenne en considération la demande du conseil municipal, et fasse droit dans la limite du possible, à une supplique justifiée par les considérations précédentes.» 
du réseau avait fait tourner la roue de la bonne fortune ferroviaire, entrainant un profond remaniement de son chapelet de dépôts entre Paris et Dijon : une "délocalisation » avant la lettre, préjudiciable à Tonnerre, bénéfique à Migennes. 